\title{
The lymph nodes draining the small intestine and colon are anatomically separate and immunologically distinct
}

\author{
SA Houston ${ }^{1}$, V Cerovic ${ }^{1}$, C Thomson ${ }^{1}$, J Brewer $^{1}$, AM Mowat ${ }^{1}$ and S Milling ${ }^{1}$
}

Dendritic cells (DCs) in the small intestine (SI) and colon are fundamental to direct intestinal immune responses; they migrate to the mesenteric lymph nodes (MLNs) and prime Tcells. We demonstrate anatomical segregation of lymphatic drainage from the intestine, specifically that DCs from the SI and colon migrate to different nodes within the MLN, here called the SMLN and cMLN. As a consequence, different frequencies of DC subsets observed in the SI and colon are reflected among the DCs in the SMLN and cMLN. Consistent with the Sl's function in absorbing food, fed antigen is presented in the SMLN, but not in the CMLN. Furthermore, the levels of expression of CCR9 and $\alpha 4 \beta 7$ are increased on Tcells in the sMLN compared with the cMLN. DCs from the cMLN and colon are unable to metabolize vitamin A to retinoic acid (RA); thus, DCs may contribute to the differential expression of tissue homing markers observed in the sMLN and CMLN. In summary, the sMLN and cMLN, and the DCs that migrate to these LNs are anatomically and immunologically separate. This segregation allows immune responses in the SI and colon to be controlled independently.

\section{INTRODUCTION}

The intestine contains more immune cells than any other organ, reflecting the fact that it is continually exposed to a plethora of dietary antigens and commensal bacteria. The largest tissues in the gastrointestinal tract are the small intestine (SI) and colon, which form physiologically and functionally distinct compartments. Although the SI is specialized for the digestion of food, the primary function of the colon is the reabsorption of water. ${ }^{1}$ The SI and colon also provide profoundly different immune environments, with the vast majority of commensal bacteria residing in the colon. ${ }^{2,3}$ The physiological differences between the SI and colon are also reflected by numerous differences in immune responses, e.g., in the induction of tolerance. Although in the SI tolerance to food antigens develops both systemically and locally, the tolerance to colonic bacteria only acts in a localized manner. ${ }^{4}$ Furthermore, different homing molecules induced by dendritic cells (DCs) direct activated T cells to migrate to the SI and colon. ${ }^{5}$ For instance, the G-protein-coupled receptor GPR15 may contribute to the homing of T cells to the colon, ${ }^{6}$ whereas the chemokine receptor CCR9 and the integrin $\alpha 4 \beta 7$ preferentially direct $\mathrm{T}$ cells to the SI and are induced in the presence of metabolites of vitamin $\mathrm{A}^{7,8}$ In addition, most diseases that affect the intestine affect specific regions. For example, ulcerative colitis is restricted to the colon, while Crohn's disease affects both the SI and colon. Despite advances in our understanding of the immunological differences between the SI and colon, ${ }^{1,9,10}$ the mechanisms controlling these differences have been largely overlooked in recent years.

Mesenteric lymph nodes (MLNs) are an important site of T-cell activation for both the SI and colon. DCs continually migrate from intestinal tissues to the MLNs where they present antigen and control the development, migration, and functional differentiation of cells of the adaptive immune system. The MLN is often assumed to be the LN draining both the SI and colon, despite the fact that studies have shown different parts of the intestine drain to distinct nodes in the MLN chain. The anatomy of lymphatic drainage from the SI and colon was first examined over 40 years ago ${ }^{11,12}$ and a more recent study has updated these findings. ${ }^{13}$ These studies show that lymphatics from different areas of the intestine drained injected dyes to distinct nodes within the chain of MLNs.

${ }^{1}$ Centre for Immunobiology, Institute for Infection, Immunity and Inflammation, College of Medical, Veterinary and Life Sciences, University of Glasgow, Glasgow, UK. Correspondence: S Milling (simon.milling@glasgow.ac.uk) 
As the SI and colon are immunologically distinct and drain to different regions of the MLNs, we hypothesized that the different nodes of the MLNs would reflect the characteristics of the different environments that influence them. We predicted therefore that migratory cells, and in particular DCs, found in the distinct mesenteric nodes, and the nodes themselves, would display different functions.
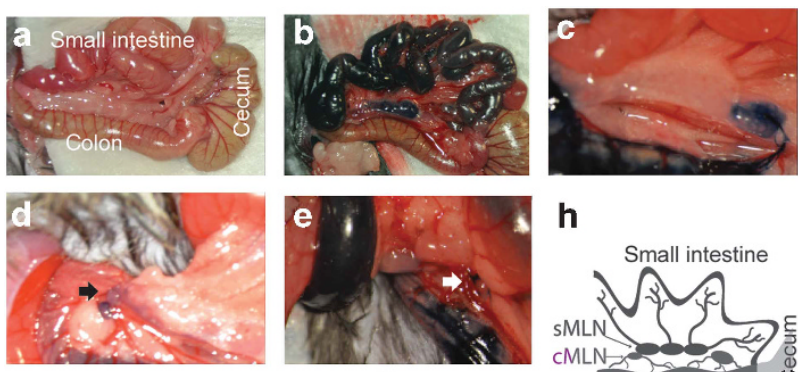

h
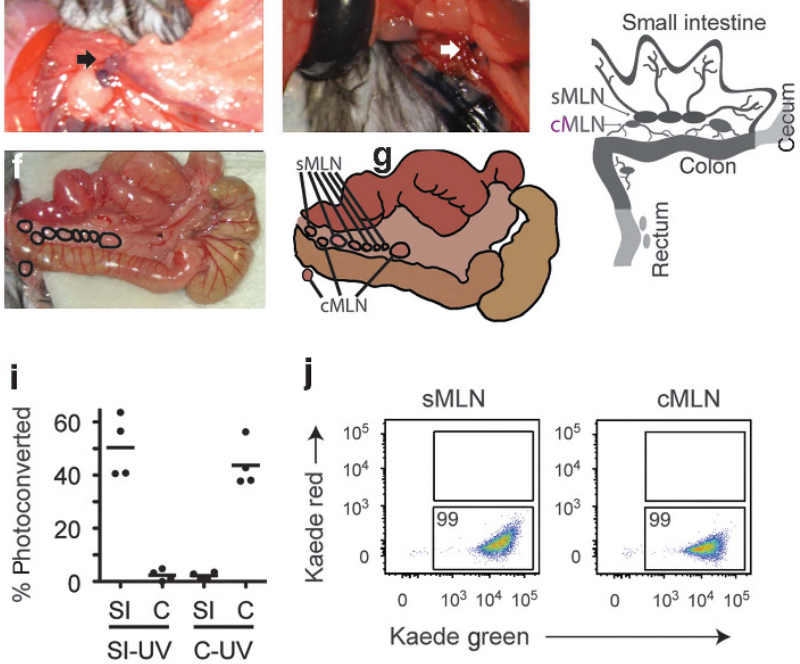

j

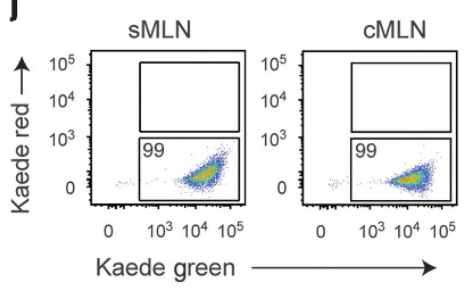

k
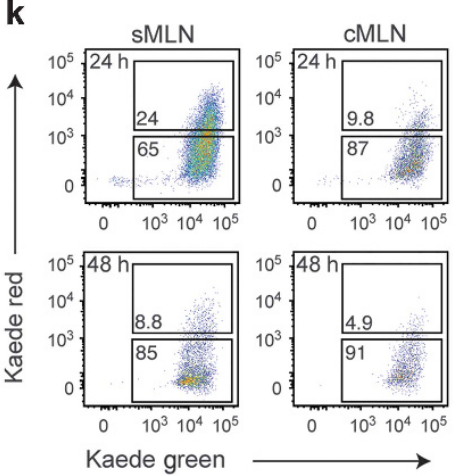

I
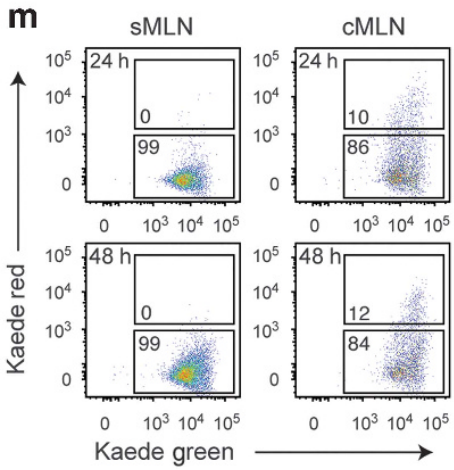

There are many features of intestinal DCs that may differ between the SI and colon, and therefore between different nodes within the mesenteric chain. Despite this, many groups, including ourselves, have previously failed to discriminate between the distinct MLN nodes when examining the populations of cells migrating from both the SI and colon. ${ }^{14,15}$ A deeper understanding of these differences would enable the most appropriate samples to be collected when examining the details of the immune responses generated in the intestine.

Here we confirm that lymph drains from different regions of the intestine to different nodes within the MLNs, and describe the anatomically distinct migration pathways of DCs from the SI and colon. In addition, we show that DCs originating from the SI induce T-cell responses in specific nodes of the MLN, and that different nodes contain $T$ cells with distinct characteristics. Our data emphasize the immunological separation of responses originating in the SI and colon, and have far-reaching implications, both for those performing analyses of intestinal immune responses and for those developing tissue-specific treatments for pathologies that affect specific regions of the intestine.

\section{RESULTS}

\section{Distinct nodes of the MLN chain drain the SI and colon}

To identify the individual nodes draining each part of the intestine we visualized and compared the lymphatic drainage from the SI and colon. To achieve this, Evans blue dye was injected beneath the subserosal layer into the intestinal wall. The dye was carried from the tissue by the afferent lymphatics, allowing direct examination of the lymphatic system and identification of the specific nodes draining the injected area (Figure 1a-e). Following injection of Evans blue into the SI, the central mesenteric nodes stained blue (Figure 1b). These nodes will be referred to hereafter as the small intestinal MLN (sMLN). When Evans blue was injected into the proximal colon, the dye reached the first node in the MLN chain, located closest to the cecum (Figure 1c). Interestingly, a second node also stained blue following injection of dye into the proximal colon. This LN was inferior to the main MLN chain, close to the abdominal aorta, and distal to the superior mesenteric vein

Figure 1 Distinct nodes of the mesenteric lymph node (MLN) chain drain the small intestine $(\mathrm{SI})$ and colon. The intestines were exposed at laparotomy (a), and Evans blue dye was injected underneath the serosa of either the SI (b), the proximal colon (c,d) or distal colon (e), or were uninjected $(\mathbf{a}, \mathbf{f})$. Five minutes later, MLNs were photographed. Arrows indicate nodes in the colonic MLN (cMLN) marked with Evans blue. The location (g) and lymphatic drainage patterns (h) of the small intestinal MLN (sMLN) and cMLN. The iliac and caudal nodes, located against the spine, adjacent to the aortic bifurcation, are represented in grey (h). At laparotomy, the SI or colon of photoconvertible Kaede mice was exposed to low-intensity violet light for $2 \mathrm{~min}$. sMLN and $\mathrm{cMLN}$ were removed either immediately $(\mathbf{i}, \mathbf{j})$ or after 24 or $48 \mathrm{~h}$ (SI: $\mathbf{k}, \mathbf{I}$ and colon: $\mathbf{m}, \mathbf{n})$. The numbers of photoconverted migratory dendritic cells (DCs) were analyzed. Controls that were not exposed to the violet light were used to define the location of the gate used to identify the Kaede-red ${ }^{+}$DCs (j). Each dot represents an individual replicate and are pooled from at least two independent experiments. Asterisks denote statistical significance $\left({ }^{\star} P<0.05\right.$ and $\left.{ }^{\star \star} P<0.01\right)$. 
(Figure 1d). Following Evans blue injection into the distal colon, the first mesenteric node to stain blue was located in the intestinal mesentery adjacent to the abdominal aorta (Figure 1e). This is likely to be the inferior mesenteric node. Collectively, these three nodes that drain the lymph from the colon will be referred to as the colonic MLN (cMLN); a diagram highlighting their positioning and a schematic representing the lymphatic drainage to the sMLN and cMLN are shown in Figure 1f-h. The iliac LNs are located adjacent to the spine, anterior of the aortic bifurcation, and were not labeled with Evans blue after any of our injections. They may drain lymph from the caudal nodes, which drain the rectum. ${ }^{12}$

To identify how the separation of the lymphatic drainage from the SI and colon influenced the destinations of the DCs migrating from the intestine, we used transgenic mice that ubiquitously express the green fluorescent Kaede protein. This protein is irreversibly converted from green to red fluorescence following exposure to low-intensity violet light. ${ }^{16,17}$ At laparotomy, all accessible parts of the SI or colon of Kaede mice were exposed to violet light for $2 \mathrm{~min}$. This resulted in the photoconversion of around $50 \%$ of all live $\mathrm{CD} 45^{+}$cells in the SI or colon immediately following violet light exposure (Figure 1i) and no photoconverted cells were detected in the sMLN or cMLN (Figure 1j). Thus, detectable photoconversion occurs in a high frequency of target cells and is restricted to the site of light exposure. Twenty-four or $48 \mathrm{~h}$ after photoconversion, the sMLN or cMLN were analyzed. Twenty-four hours after exposure of the SI to violet light, significantly more photoconverted Kaede-red DCs were detected in the sMLN than in the cMLN (Figure 1k,l). The Kaede-red DCs were all found among the population of migratory $\mathrm{MHCII}^{\mathrm{hi}} \mathrm{CD} 11 \mathrm{c}^{+}$DCs (Supplementary Figure S1 online). Fewer Kaede-red DCs were present in the sMLN $48 \mathrm{~h}$ after illuminating the SI, but there were still significantly more Kaede-red DCs in the sMLN than in the cMLN. Conversely, following the exposure of the colon to violet light, Kaede-red cells were found almost exclusively in the cMLN but not in the sMLN after 24 and $48 \mathrm{~h}$ (Figure 1 $\mathbf{m}, \mathbf{n}$ ). In some experiments, violet light exposure was confined to smaller regions of the SI or colon, revealing that photoconversion of specific regions of the SI or colon reproducibly results in significant enrichment of Kaede-red DCs in individual nodes of the sMLN or cMLN (Figure 2). Thus, lymph and DCs that originate in the SI drain to the sMLN, whereas lymph and DCs from the colon drain to the cMLN.

\section{Differences between DC populations in the SI and colon are reflected in the sMLN and $\mathrm{CMLN}$}

We and others have observed striking differences between the DC populations in the mucosa of the SI and colon. ${ }^{18}$ Therefore, we sought to determine whether the composition of migratory DC subsets in the sMLN and cMLN reflected the corresponding mucosal sites. As we found previously, DCs in the SI and colon could be divided into four subsets based on their expression of CD103 (integrin $\alpha \mathrm{E}$ ) and CD11b. ${ }^{18}$ In the SI the predominant population of DCs was the $\mathrm{CD} 103{ }^{+} \mathrm{CD} 11 \mathrm{~b}^{+} \mathrm{DCs}$, but these cells were rare in the colon (Figure 3a). The sMLN had the highest proportion of $\mathrm{CD} 103^{+} \mathrm{CD} 11 \mathrm{~b}{ }^{+} \mathrm{DCs}$, whereas the $\mathrm{CD}_{103}{ }^{+} \mathrm{CD} 11 \mathrm{~b}^{-} \mathrm{DCs}$ and CD103 ${ }^{-} \mathrm{CD} 11 \mathrm{~b}^{+}$DCs were more numerous in the cMLN (Figure $3 \mathbf{b}$ ), reflecting the relative frequencies of these DC populations in the colon. Interestingly, there appeared to consistently be lower CD103 expression on DCs from the sMLN and CMLN when compared with the corresponding intestinal tissue sample. This might reflect a downregulation of CD103 expression on arrival in the LN.

We observed that there were no significant differences between the sMLN/SI or cMLN/colon in the levels of expression of $\operatorname{SIRP} \alpha$, which was present on $\mathrm{CD}_{11} \mathrm{~b}^{+} \mathrm{DCs}$ in both the SI and colon, ${ }^{18,19}$ nor were there any differences in the expression of XCR1 or CD8 $\alpha$, present on the CD103 ${ }^{+}$ $\mathrm{CD}_{11 \mathrm{~b}}{ }^{-}$DC subset, ${ }^{15}$ between the SI and colon sMLN or cMLN. There were also no significant differences in the levels of expression of co-stimulatory molecules CD80 and CD86 between the sMLN/SI and the cMLN/colon (data not shown).

Previously, we have shown that the $\mathrm{CD}_{103}{ }^{-} \mathrm{CD}_{11 \mathrm{~b}}{ }^{+} \mathrm{DCs}$ migrating in pseudo-afferent lymph express intermediate levels of CX3CR $1 .{ }^{18}$ Using CX3CR $1^{\mathrm{GFP} /+}$ mice $^{20}$ we now compared the expression of CX3CR1-GFP on DC subsets from the SI and colon, and from the sMLN and cMLN (Figure 3c). Consistent with our previous data, we observed that $\mathrm{CD} 103^{-} \mathrm{CD} 11 \mathrm{~b}^{+}$ DCs express intermediate levels of CX3CR1 in the SI, colon, sMLN, and cMLN. Interestingly, we also detected a previously unreported expression of CX3CR1-GFP in $\mathrm{CD}_{103}{ }^{+} \mathrm{CD} 11 \mathrm{~b}{ }^{+}$ colonic DCs. A significantly higher percentage of $\mathrm{CD}_{103}{ }^{+} \mathrm{CD}_{11 \mathrm{~b}}{ }^{+} \mathrm{DCs}$ in the colon and cMLN expressed CX3CR1-GFP than in either the SI or sMLN. Our previous studies have also shown that DCs migrating in pseudo-afferent lymph express low levels of the eosinophil marker SiglecF. ${ }^{18}$ In contrast to CX3CR1, CD $103^{+} \mathrm{CD}_{11 \mathrm{~b}}{ }^{+}$DCs from the SI and sMLN expressed more SiglecF than $\mathrm{CD}_{103}{ }^{+} \mathrm{CD}_{11 \mathrm{~b}}{ }^{+} \mathrm{DCs}$ from either the colon or cMLN (Figure 3d). Thus, differences between DCs in the SI and colon, which may result from their exposure to these different tissue environments, are reflected in the DC populations in the draining sMLN and cMLN.

\section{Antigen presentation occurs independently in the sMLN and $\mathrm{CMLN}$}

To examine the functional consequences of the anatomical compartmentalization of DC migration from the SI and colon, we investigated whether antigen acquired in the SI was preferentially presented by DCs in the sMLN. Soluble ovalbumin (OVA) was fed to mice, which had previously been adoptively transferred with carboxyfluorescein succinimidyl ester (CFSE)-labeled $\mathrm{T}$ cell receptor-transgenic OVA-specific $\mathrm{CD}^{+}$OT-II $\mathrm{T}$ cells. Three days later, we observed significantly more proliferating $\mathrm{T}$ cells in the sMLN compared with the cMLN (Figure 4a), or inguinal LNs used as a non-intestinal control. To extend these findings to $\mathrm{CD} 8{ }^{+}$ $\mathrm{T}$-cell priming with endogenous antigen, we used IFABP-tOVA 232-4 (232-4) mice that express a cytoplasmic form of OVA exclusively in mature SI enterocytes ${ }^{21}$ (Figure 4b). CD103 ${ }^{+}$ $\mathrm{CD}_{11 \mathrm{~b}}{ }^{-}$migratory DCs are the only cells able to cross-present OVA from these mice ${ }^{15}$ and therefore we co-cultured CD103 ${ }^{+}$ 

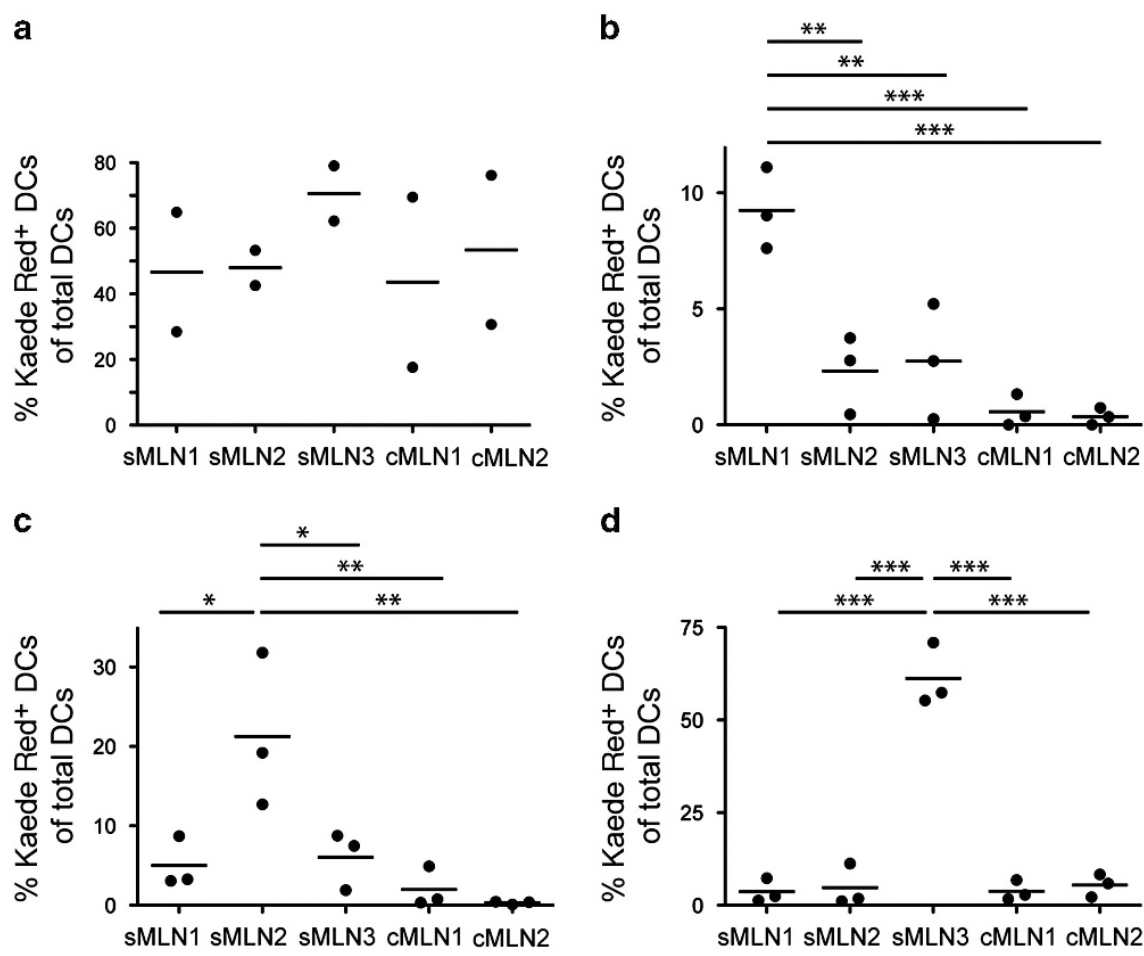

d

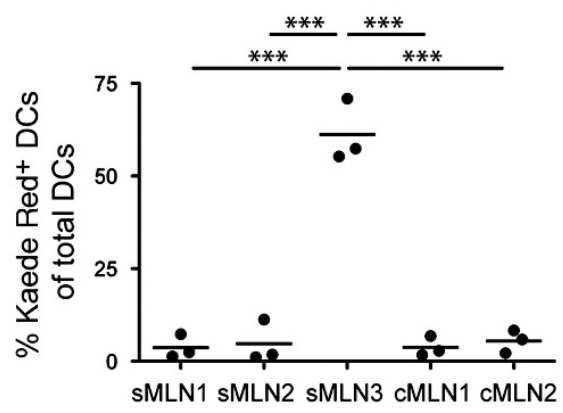

e
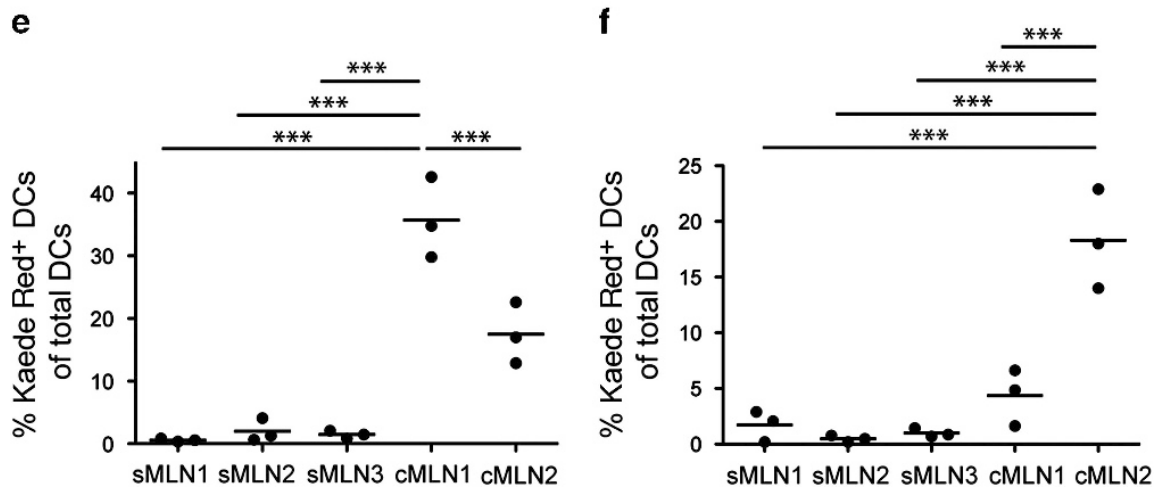

Figure 2 Dendritic cells (DCs) from specific regions within the small intestine (SI) and colon migrate preferentially to specific nodes within the small intestinal mesenteric lymph node (sMLN) or colonic MLN (cMLN). At laparotomy, regions corresponding approximately either to the duodenum (sMLN1), jejunum (sMLN2), ileum (sMLN3), proximal (cMLN1), or distal (cMLN2) colon of photoconvertible Kaede mice were exposed to low-intensity violet light for $2 \mathrm{~min}$. Immediately afterwards, single-cell suspensions were prepared from the intestinal tissues of two mice, to confirm the photoconversion procedures had been successful (a). After $24 \mathrm{~h}$, individual nodes of the sMLN and cMLN were removed from the remaining animals (b-f). The proportions of photoconverted migratory DCs were analyzed. Each dot represents an individual animal. sMLN and CMLN nodes are numbered independently, in ascending order, starting at the stomach. Asterisks denote statistical significance $\left({ }^{*} P<0.05\right.$ and $\left.{ }^{* *} P<0.01,{ }^{* *} P<0.001\right)$.

$\mathrm{CD}_{11 b^{-}}$DCs from the sMLN and cMLN of 232-4 mice with naive CFSE-labeled OVA-specific OT-I CD8 ${ }^{+}$T cell receptortransgenic $\mathrm{T}$ cells (Figure $\mathbf{4 b}$ ). Only DCs isolated from the sMLN presented the SI-derived OVA, inducing proliferation of the naive T cells (Figure 4c), whereas DCs isolated from the cMLN could not do this. We observed this to be true at all ratios of DCs to T cells, from 1:8 down to 1:64 (data not shown).

\section{The frequencies of FoxP3 ${ }^{+} \mathrm{T}$ cells and GPR15 levels are higher in the colon}

We observed a higher frequency of FoxP ${ }^{+} \mathrm{T}$ cells in the colonic lamina propria (LP) compared with the SI LP. ${ }^{22}$ However, we did not observe significant differences between the percentages of FoxP3 ${ }^{+}$T cells between the sMLN or cMLN (Figure 5a,b). To investigate whether different mechanisms of cell migration to the colon may be responsible for the different levels of $\mathrm{FoxP}^{+} \mathrm{T}$ cells in the colon, we examined $\mathrm{CD}^{+}{ }^{+} \mathrm{CD} 4{ }^{+} \mathrm{T}$ cells isolated from the sMLN, SI, cMLN, and colon for expression of mRNA for the G-protein-coupled receptor GPR15. Recent work has indicated that GPR15 has a role in leukocyte homing to the colon. ${ }^{6}$ The authors used a transgenic GPR15-GFP knock-in mouse to show that under homeostatic conditions GPR15 was selectively expressed by colonic regulatory $\mathrm{T}$ cells (Tregs). Therefore, we sought to determine whether there were differences in the expression of GRP15 in CD4 ${ }^{+}$T cells between the sMLN and cMLN. In line 
a

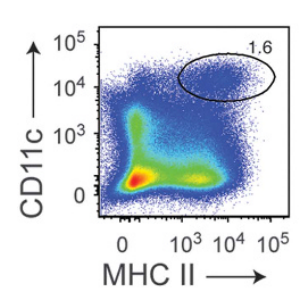

b

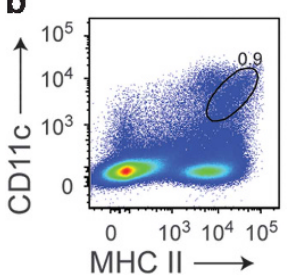

c

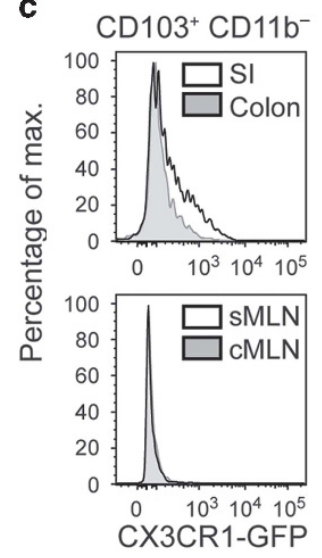

d

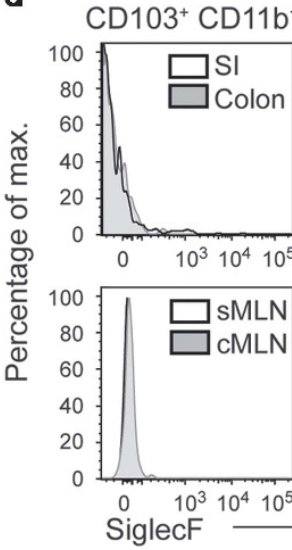

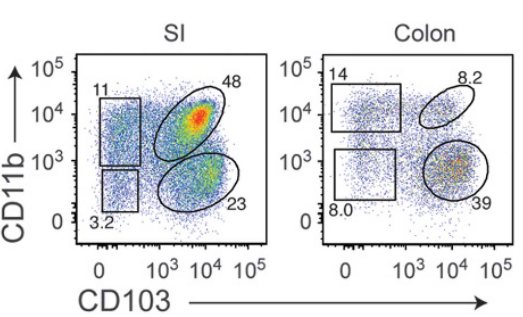
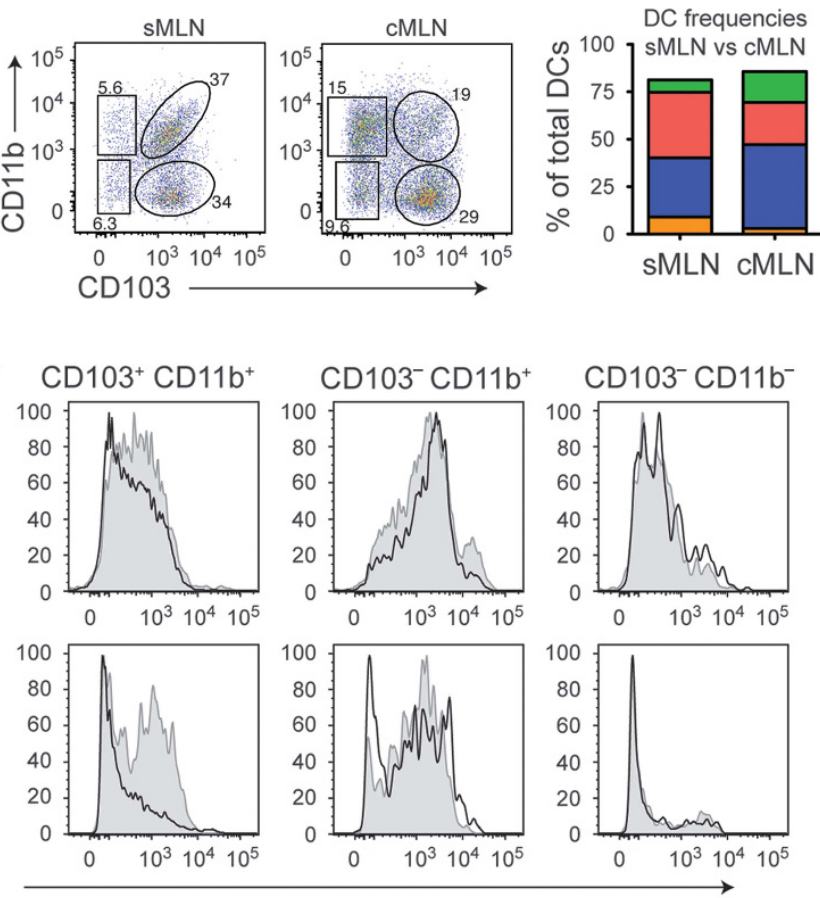

$\mathrm{CD}^{-} \mathrm{C}^{-} \mathrm{CD} 11 \mathrm{~b}^{+}$

$\mathrm{CD} 103^{+} \mathrm{CD} 11 \mathrm{~b}^{+}$

$\mathrm{CD}^{2} 3^{+} \mathrm{CD} 11 \mathrm{~b}^{-}$

$\mathrm{CD}^{\circ} \mathrm{C}^{-} \mathrm{CD} 11 \mathrm{~b}^{-}$
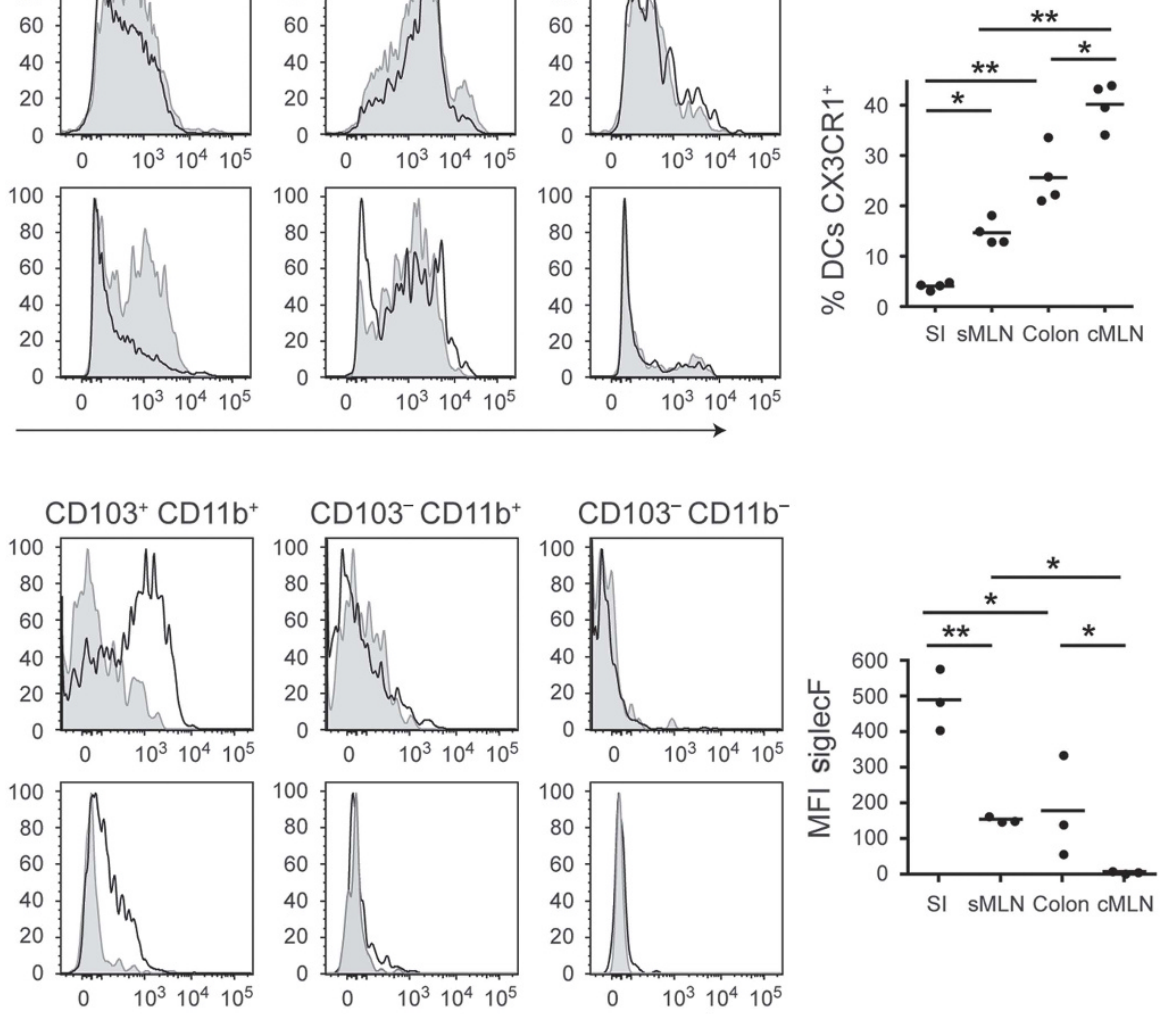

Figure 3 Differences between dendritic cell (DC) populations in the small intestine (SI) and colon are reflected in the small intestinal mesenteric lymph node (sMLN) and colonic MLN (cMLN). DCs from the SI and colon (pregated as live, CD45 ${ }^{+} \mathrm{B}_{220^{-}} \mathrm{CD}^{-} 4^{-} \mathrm{MHCII}^{\mathrm{hi}} \mathrm{CD} 11 \mathrm{c}{ }^{+}$) $(\mathbf{a})$ or SMLN and cMLN (pregated as live, $\mathrm{CD} 45^{+} \mathrm{B} 220^{-} \mathrm{CD} 64^{-} \mathrm{MHCII}^{\text {hi }} \mathrm{CD} 11 \mathrm{c}^{+}$) (b) were assigned to subsets based on their expression of CD11b and $\mathrm{CD} 103$. The expression of CX3CR1 (c) or SiglecF (d) was analyzed on each DC subset from the SI, colon, sMLN, and cMLN of CX3CR $1^{\mathrm{gfp} /+}$ mice or following staining of SiglecF in wild-type (WT) mice. Histograms show the level of expression of CX3CR1-GFP or SiglecF on each subset. The graph compares the level of expression of CX3CR1 or SiglecF on $\mathrm{CD}_{103}{ }^{+} \mathrm{CD} 11 \mathrm{~b}{ }^{+} \mathrm{DC}$ from all four tissues. Data are presented as means and are pooled from two independent experiments. Asterisks denote statistical significance $\left({ }^{\star} P<0.05\right.$ and $\left.{ }^{* *} P<0.01\right)$.

with the previous studies, we found that $\mathrm{CD} 4^{+} \mathrm{T}$ cells from the colonic LP expressed around 40 times more GPR15 than those in the SI. Surprisingly, however, there were no differences in GPR15 expression by CD4 ${ }^{+}$T cells from the sMLN and cMLN, with very low levels observed in both (Figure 5c). To investigate these Tregs more closely, we examined expression of neuropilin-1, to distinguish natural from induced Tregs. ${ }^{23}$ In contrast to the result with GPR15, there was a significantly 
a
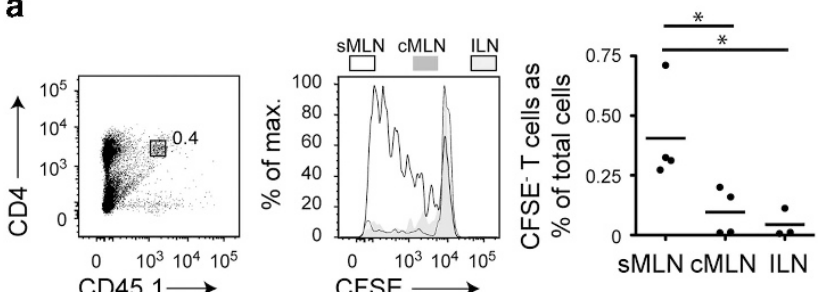

b
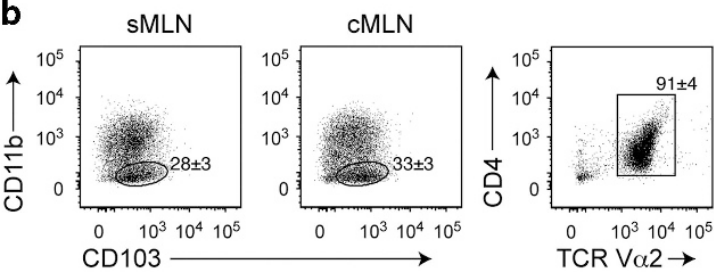

C
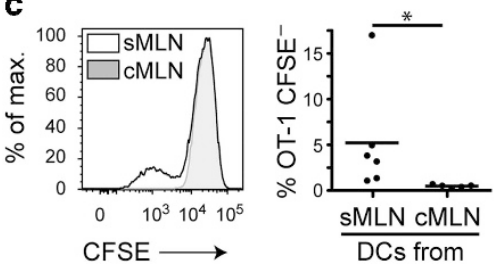

Figure 4 Antigen presentation occurs independently in the small intestinal mesenteric lymph node (sMLN) and colonic MLN (cMLN). (a) Carboxyfluorescein succinimidyl ester (CFSE)-labeled CD45.1 OT-II cells $\left(5 \times 10^{6}\right)$ were transferred intravenously to CD45.2 hosts. Twenty-four hours later, the animals received $10 \mathrm{mg}$ ovalbumin (OVA) by oral gavage. Three days after OVA delivery, the SMLN and CMLN were removed and frequencies of $\mathrm{CFSE}^{-} \mathrm{CD} 45.1^{+}$cells of total cells were compared. The graph shows data from four mice. (b) Cross-presenting CD103 ${ }^{+}$CD $11 b^{-}$ dendritic cells (DCs) were purified from sMLN and CMLN of 232-4 mice, which express OVA only in the epithelium of the small intestine (SI). DCs were cultured at a 1:8 DC:T cell ratio with $10^{5} \mathrm{CD} 62 \mathrm{~L}^{+} \mathrm{CD} 8 \alpha^{+}$CFSElabeled OT-I T cells. Numbers on dot plots show the percentage of cells in the gate \pm s.e.m. (c) Levels of proliferation were analyzed 3 days later as shown in the histogram. The graph shows data from 11 mice, from 2 independent experiments. Asterisks denote statistical significance $\left({ }^{\star} P<0.05\right)$.

lower frequency of neuropilin- $1^{+} \mathrm{T}$ cells in the colon than in the SI (Figure 5d). There was no significant difference in the frequencies of neuropilin-1 between the sMLN and cMLN. We also examined expression of the IL-33 receptor ST-2 on T cells. ST-2 has recently been reported to be preferentially expressed on colonic Tregs. ${ }^{24}$ As expected, we found ST-2 ${ }^{+} \mathrm{T}$ cells in the colon (Figure 5e); however, these were not present at significantly higher frequencies in the cMLN than in the sMLN.

\section{RALDH activity and CCR9 expression differ between the SI/sMLN and the colon/cMLN}

One of the characteristic features of DCs in the SI LP is the production of the vitamin A metabolite retinoic acid (RA), which is responsible for their ability to induce CCR9 expression on $\mathrm{T}$ cells and for at least part of their capacity to generate FoxP3 ${ }^{+}$Treg. ${ }^{7,25-27}$ Although it has been shown that $\mathrm{CD} 103^{+}$ DC from MLNs also produce RA, ${ }^{26}$ it is not known how this activity might be compartmentalized between the sMLN and cMLN. Therefore, we examined the activity of aldehyde dehydrogenase, which is required for the generation of RA, in DCs from the SI and colonic LP, sMLN, and cMLN. We observed that DCs from the SI and sMLN had significantly higher aldehyde dehydrogenase activity than DCs from the colon and cMLN (Figure 6a). Among the DC subsets, it was the $\mathrm{CD}_{103}{ }^{-} \mathrm{CD} 11 \mathrm{~b}^{+}$DCs that displayed the greatest difference in aldehyde dehydrogenase activity between the colon and SI (Figure 6b). In parallel, there were significantly more CCR9 ${ }^{+}$ and $\beta 7^{+}$T cells both in the SI LP than in the colonic LP, and in the sMLN than in the cMLN (Figure 6c,d).

These data indicate that colonic DCs may not be influenced by the immunomodulatory effects of dietary agents such as vitamin A.

\section{DISCUSSION}

The SI and colon represent vastly different immunological niches; therefore, the cells that migrate from the SI or colon are likely to have been exposed to enormously different stimuli. As DCs are essential for the induction of an adaptive immune response, acting as a bridge between the innate and adaptive immune system we compared lymphatic drainage from the SI and colon, and its effects on DC migration. Here we demonstrate that in the steady state there is anatomical segregation of cell migration between the SI and colon to specific regions of the MLNs. These distinct parts of the MLNs can be examined separately, to allow better analysis of the immune responses in the SI and colon.

To more accurately identify which parts of the intestine supply lymph to each of the nodes of the MLN chain, we examined the lymphatic drainage from the SI and colon by the injection of Evans blue dye. This allowed direct examination of the intestinal afferent lymphatic system and the MLNs that drain the intestine. Following the subserosal injection of Evans blue into the SI, the central nodes of the MLN chain stained blue, identifying them as the sMLNs. This phenomenon has previously been observed, with the identification of the middle MLNs draining the jejunum and ileum. ${ }^{9,11-13}$ Conversely, when Evans blue was subserosally injected into the colon, the most distal node in the main MLN chain was labeled. This was consistent with previous reports in mice and rats. ${ }^{11,12}$ However, this was not the only node to drain the colon. Interestingly, a second, previously unreported, node at the opposite end of the MLN chain was also found to be draining the colon. In addition, as previously reported, following the labeling of the distal colon, an LN distinct from the main MLN chain located in the intestinal mesentery adjacent to the abdominal aorta was found to drain the most distal colon. ${ }^{12}$ The anatomical segregation of lymphatic drainage between the SI and colon has previously been observed in mice and rats but recently many groups, including ourselves, have overlooked this.

As antigen can be carried to the MLNs by migratory DCs, which contribute to determining the outcome of the immune response, we decided to investigate DC migration to the MLN. Therefore, we explored the anatomical segregation of lymphatic drainage from the SI and colon in the steady state using Kaede mice. Kaede mice have previously been used to monitor DC 

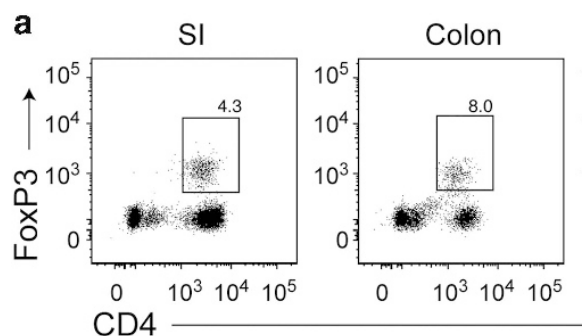
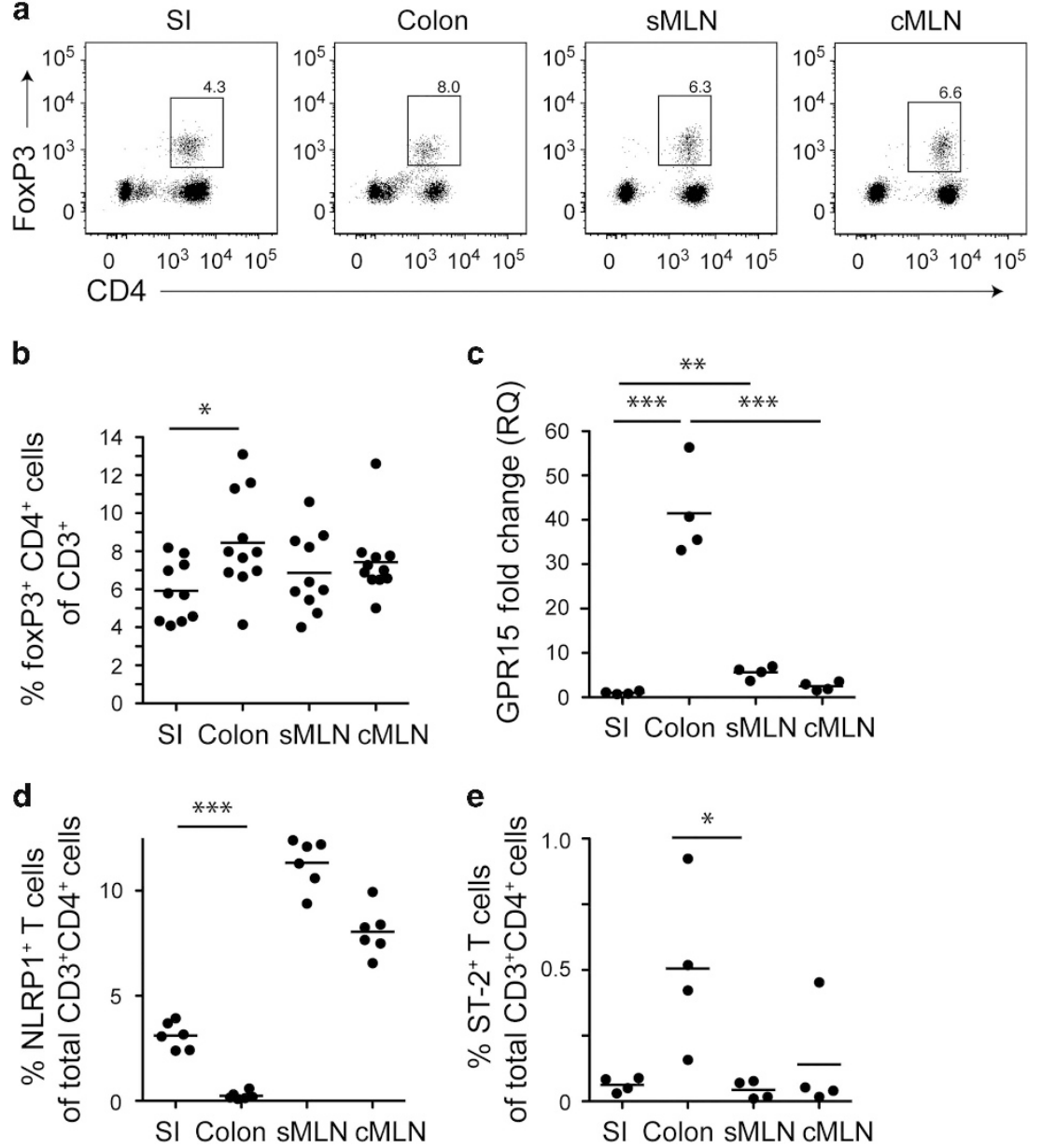

Figure 5 The frequencies of FoxP3 ${ }^{+}$T cells and GPR15 levels are increased in the colon. The frequency of neuropilin (NLRP)-1 ${ }^{+}$cells is higher in the small intestine (SI). Cells from the SI, colon, small intestinal mesenteric lymph node (sMLN), and colonic MLN (cMLN) were stained for the expression of FoxP3, NLRP-1, and ST-2. Fluorescence-activated cell sorted (FACS) plots show representative examples of FoxP3 and CD4 staining from each tissue pregated on live, $\mathrm{CD}_{4} 5^{+}, \mathrm{CD} 3^{+}$cells $(\mathbf{a})$. The graph (b) shows the proportion of FoxP3 ${ }^{+} \mathrm{T}$ cells in individual mice. $\mathrm{CD} 3^{+} \mathrm{CD} 4^{+} \mathrm{T}_{\text {cells }}$ were purified by FACS sorting and quantitative real-time PCR was used to determine the relative expression of GPR15, normalized to the housekeeping gene TBP (c). Graphs (d,e) show the proportion of NRP-1 ${ }^{+}$or ST-2 ${ }^{+}$T cells in individual mice $\left({ }^{\star} P<0.05,{ }^{* \star} P<0.01\right.$, and $\left.{ }^{* \star *} P<0.001\right)$.

migration in peripheral LNs, ${ }^{16}$ as well as B-cell migration in the intestine. ${ }^{17}$ However, intestinal DC migration has not been investigated using this system. To analyze the migration of intestinal DCs, mice underwent the photoconversion of either the SI or colon, and the migration of photoconverted Kaede-red DCs was compared. Following the photoconversion of the SI, Kaede-red DCs were found in the sMLN. Conversely, following the photoconversion of the colon, Kaede-red DCs were found in the cMLN. Consistent with results from Evans blue experiments, the Kaede data show that the anatomy of the afferent lymphatics dictates that DCs that originate in the SI drain to the sMLNs, while DCs that originate in the colon drain to the cMLNs.

Intestinal DCs can be separated into four functionally distinct subsets based on the expression of CD11b and CD103. ${ }^{18}$ Previous work has shown differences in the frequencies of the DC subsets between the SI and colon. ${ }^{28}$ In the SI, the most numerous population of DCs is the IRF4-dependent $\mathrm{CD}_{103}{ }^{+} \mathrm{CD} 11 \mathrm{~b}^{+}$subset, followed by the
BatF3- and IRF8-dependent $\mathrm{CD}_{103}{ }^{+} \mathrm{CD} 11 \mathrm{~b}^{-}$population. The two $\mathrm{CD}_{103}{ }^{-}$DC populations were also identified. The characteristics of the DCs in the colon have been less well documented. ${ }^{29}$ Although all four subsets of DCs were identified in the colon, they were present at different frequencies than in the SI. The $\mathrm{CD} 103^{+} \mathrm{CD} 11 \mathrm{~b}{ }^{+}$DC subset was a much smaller component of the colonic DC population than in the SI. The largest component in the colon was the $\mathrm{CD}_{103}{ }^{+} \mathrm{CD} 11 \mathrm{~b}^{-}$ population, which is able to efficiently cross-present selfantigen. ${ }^{15}$ Following the photoconversion of the SI or colon in Kaede mice, migratory DCs could be identified in the MLN by their expression of the Kaede-red protein. All the Kaede-red DCs were found in the MHICII ${ }^{\text {hi }} \mathrm{CD} 11 \mathrm{c}^{+}$gate, never in the $\mathrm{MHCII}^{+} \mathrm{CD}_{11 \mathrm{c}^{\text {hi }}}$ DC gate (Supplementary Figure S1). This confirms earlier reports that migratory DCs in the MLNs have higher expression of $\mathrm{MHCII},{ }^{30}$ similar to migratory DCs in the skin and lung. ${ }^{31}$ In the sMLN and cMLN, we identified different proportions of the migratory DC subsets, reflecting the proportions of the subsets in the source tissue. 
a

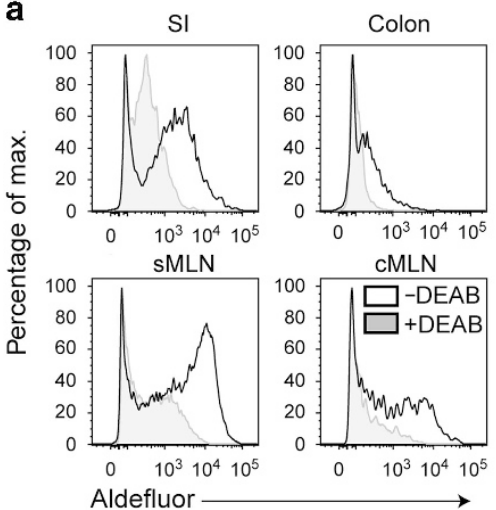

C

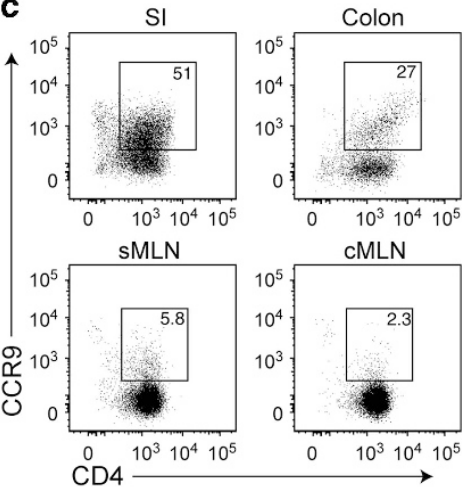

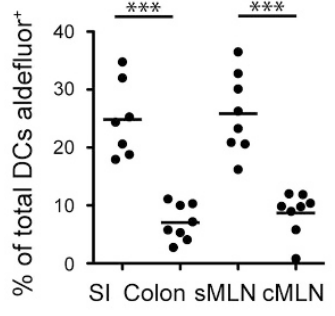

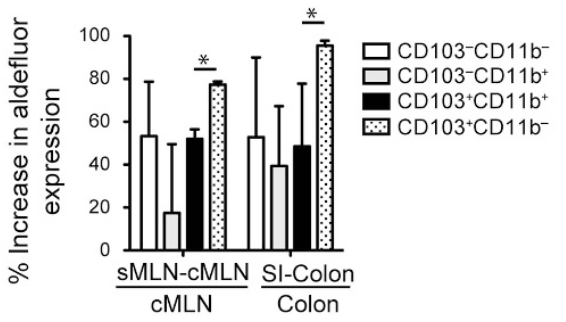

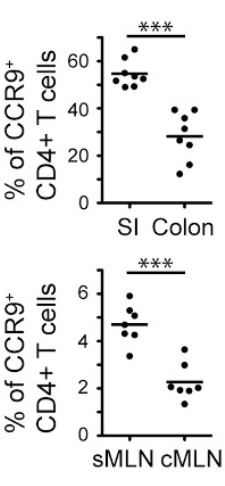

d
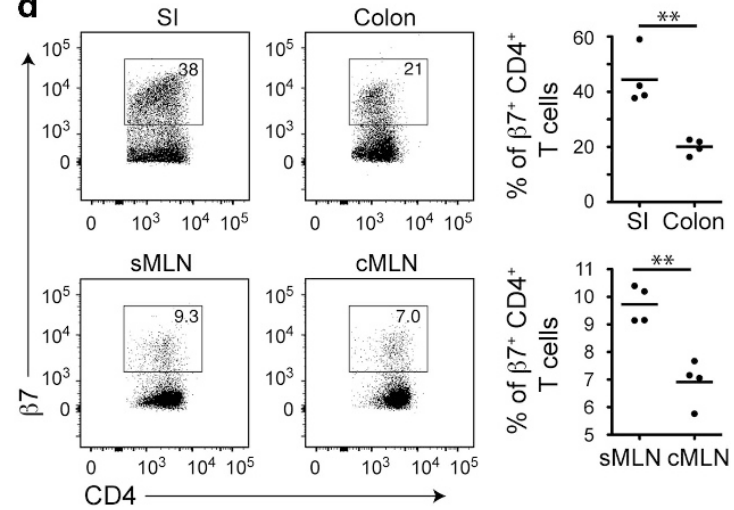

Figure 6 RALDH activity CCR9 and $\beta 7$ expression differ between the small intestine (SI)/small intestinal mesenteric lymph node (sMLN) and the colon/ colonic MLN (cMLN). (a) Cells from the SI, colon, sMLN, and cMLN were incubated with aldefluor in the presence or absence of its inhibitor DEAB and analyzed by flow cytometry. Histograms show representative examples of staining from each tissue. The graph shows the \% of aldefluor-positive cells (\% of positive ' - DEAB cells' - \% of ' + DEAB cells') for the SI, colon, sMLN, and cMLN from eight mice. (b) Dendritic cell (DC) subsets from the SI, colon, SMLN, and cMLN were analyzed for aldefluor activity and the percentage increases between the colon and SI or CMLN and SMLN were compared. CCR9 (c) and $\beta 7$ (d) expression was analyzed on $\mathrm{CD} 4{ }^{+} \mathrm{T}$ cells from SI, colon, sMLN, and cMLN. Dot plots show representative data. Graphs show analysis of results from 14 mice, pooled from 2 independent experiments. Asterisks denote statistical significance $\left({ }^{\star} P<0.05\right.$, ${ }^{* *} P<0.01$, and $\left.{ }^{* * *} P<0.001\right)$.

In the sMLN and SI the $\mathrm{CD} 103^{+} \mathrm{CD} 11 \mathrm{~b}^{+}$DCs were the most numerous, whereas in the cMLN and colon the CD103 ${ }^{+}$ $\mathrm{CD}_{11 \mathrm{~b}}{ }^{-}$DCs were the largest population.

We have previously shown that migratory $\mathrm{CD} 103^{-} \mathrm{CD} 11 \mathrm{~b}^{+}$ DCs express intermediate levels of CX3CR1-GFP, and that F4/ 80-positive cells from the intestinal lamina propria express higher levels of CX3CR1. ${ }^{18}$ Others have suggested that CX3CR $1{ }^{\text {hi }}$ cells may migrate to the MLNs. ${ }^{32}$ Here we compared levels of CX3CR1 expression between DC subsets in the SI and sMLN, and the colon and cMLN. Under no circumstances did we identify any DCs expressing high levels of CX3CR1. Surprisingly, we found significantly higher expression of CX3CR1-GFP on $\mathrm{CD}_{103}{ }^{+} \mathrm{CD} 11 \mathrm{~b}^{+}$DCs in the colon and cMLN compared with the SI and sMLN. The differences between the levels of expression of CX3CR1 among $\mathrm{CD}_{103}{ }^{+} \mathrm{CD} 11 \mathrm{~b}{ }^{+}$DCs in the SI and sMLN compared with the colon and cMLN may reflect differences in the local environment in the different tissues.

We analyzed the functional consequences of the anatomical segregation of migrating intestinal DCs by examining whether antigens acquired in SI were presented only in the sMLN. Although the exact mechanisms controlling uptake of soluble antigen are unclear, DCs in the LP are able to acquire soluble antigen. ${ }^{33}$ As the SI is specialized for the uptake of soluble antigen, we predicted that orally administered OVA would be preferentially presented in the sMLN. After adoptive transfer of OVA-specific CD4 ${ }^{+}$OT-II T cells, mice were fed with OVA, and 3 days later proliferation of the OT-II T cells was examined in the sMLN and cMLN. As predicted, T-cell proliferation was only detected in the sMLN. To further examine whether tissuespecific antigen is differentially presented in the sMLN and cMLN, we used transgenic 232-4 mice that express cytoplasmic OVA only in mature SI enterocytes. ${ }^{21}$ DCs purified from the 232-4 sMLNs induced proliferation of OT-I T cells in vitro, whereas DCs from the cMLNs were unable to induce the proliferation of OT-I T cells. These results demonstrate that antigen acquired in the SI is initially only presented to T cells in the sMLNs. Therefore, there is not only anatomical segregation of the lymphatic drainage from the SI and colon in the steady state, but the sMLN and cMLN are also functionally separate; antigen expressed in the SI causes proliferation of naive T cells in the sMLNs. Therefore, under most circumstances, it is beneficial to analyze immune responses in these distinct MLNs separately.

RA is reported to enhance transforming growth factor- $\beta$ dependent naive T-cell conversion to FoxP3 T cells, while suppressing transforming growth factor- $\beta$-dependent Th17- 
cell differentiation. ${ }^{34}$ However, we observed no differences in the frequencies of FoxP3 ${ }^{+} \mathrm{T}$ cells between the SI, sMLN, or cMLN, while the percentage of FoxP3 ${ }^{+}$T cells in the colon was significantly higher. We tested whether this increase in FoxP3 ${ }^{+}$ $\mathrm{T}$ cells in the colon was associated with expression of GPR15. GPR15 has been reported to be a colon-homing marker for FoxP ${ }^{+}$cells, with its expression dependent on the gut microbiota and transforming growth factor- $\beta$ expression in mice. ${ }^{6}$ However, its role in human colon is less well defined. ${ }^{35}$ We observed a 40-fold increase in the level of expression of GPR15 in the colonic T cells compared with the SI; however, there was no corresponding difference between the level of GPR15 between $\mathrm{T}$ cells in the sMLNs and cMLNs. The mechanism by which GPR15 mediates colon homing of T cells is therefore not clear. The upregulation of GPR15 within a LN and subsequent migration to the colon may be a rapid process, and therefore the detection of GPR15-expressing T cells in the MLNs may be challenging. Understanding how T cells home to the colon is a matter of immunological importance and deserves further investigation.

DCs not only stimulate the proliferation of naive T cells but also induce the expression of the gut homing markers CCR9 and $\alpha 4 \beta 7$ on the responding T cells, via the actions of RA. ${ }^{7,26,27}$ $\mathrm{RA}$ is derived from dietary vitamin A via the action of retinal dehydrogenase, which can be measured using the aldefluor assay. DCs from the SI and sMLN had higher aldefluor activity than DCs from the colon and cMLN. Previous studies have shown DCs in the colon to have lower retinal dehydrogenase activity than DCs from the SI and MLN. ${ }^{26,29}$ However, these studies did not differentiate between the sMLN and cMLN. Here we observe that the differences in aldefluor expression in the SI and colon are reflected in the sMLN and cMLN. These differences were also reflected in the levels of CCR9 and $\alpha 4 \beta 7$ expressed by $\mathrm{CD}^{+}{ }^{+} \mathrm{T}$ cells in the sMLN, cMLN, SI, and colon. In the steady state, there were fewer CCR9 ${ }^{+}$and $\beta 7^{+}$T cells in the colon than in the SI, and fewer in the cMLN than in the sMLN, consistent with the idea that lack of retinal dehydrogenase activity in DCs from the cMLN and colon contributes to the lower expression of these SI-homing molecules in these tissues. As we found an increased frequency of FoxP ${ }^{+} \mathrm{T}$ cells in the colon compared with the SI, but reduced aldehyde dehydrogenase in the colon, this indicates that FoxP3 ${ }^{+} \mathrm{T}$ cells in from the SI and colon are likely to be generated by different mechanisms. These data may indicate that colonic DCs are influenced less by the immunomodulatory effects of dietary agents and are therefore functionally fundamentally different to SI DCs.

These basic differences between DCs from the SI and colon are likely to reflect the necessary anatomical compartmentalization of the immune response required between the SI and colon. DCs from the SI and colon are influenced by different factors. Although DCs from the SI are influenced by dietary agents, its likely that colonic DCs located adjacent to the major microbial burden are more likely to be influenced by microbial agents. This disassociation of the immune response between the SI and colon is likely to be beneficial, enabling responding $\mathrm{T}$ cells to be directed to the most appropriate intestinal compartment.

We here describe the anatomical segregation between DCs and antigen migrating from the SI and colon in the steady state, and provide data demonstrating that independent analyses of the sMLNs and cMLNs enable a more refined understanding of intestinal immune responses. By separately analyzing the appropriate nodes of the mesenteric chain, we have identified changes in cell populations that have previously been missed. This non-overlapping lymphatic drainage from the SI and colon to distinct parts of the MLN provides a mechanism by which immune responses in these functionally, anatomically, and immunologically specialized tissues can be independently controlled.

\section{METHODS}

Animals. C57/B16 mice were purchased from Harlan (Bicester, UK) and maintained in individually ventilated cages. OT-I, OT-II (originally from Professor Dan Littman), 232-4 IFABP-tOVA mice (originally from Professor Leo LeFrancois), Kaede (originally from Dr Kenji Kabashima), and CX3CR1 GFP/+ mice (originally from Professor Steffen Jung) were bred and maintained under specific pathogen-free conditions at the Central Research Facility, Glasgow, UK. All procedures were approved by the local ethical committee and were conducted under licenses issued by the UK Home Office.

Surgical procedures. Animals were maintained under inhalation anesthesia with isoflurane (Abbott Animal Health, Abbott Park, IL). Following laparotomy, photoconversion of either the SI or colon was performed. This technique was adapted from an established protocol. ${ }^{17}$ For SI or colon photoconversion, the intestine was exposed, the Peyer's patches and any other tissues were protected from light using aluminum foil or black card, and a 2- to $3-\mathrm{cm}$ section of the SI or colon was exposed to low power UV light ( $395 \mathrm{~nm}$, Amazon, Seattle, WA) for 2-3 min. The intestine was then replaced, the muscle layer sutured, and the skin closed using surgical clips. For the injections of Evans blue dye, procedures were performed under terminal anesthesia. The intestine was held gently with fine forceps and Evans blue dye (2\% solution, Sigma Aldrich, St Louis, MI), up to $30 \mu \mathrm{l}$ per injection site, was injected using a micro-fine syringe (30G BD Biosciences, Franklin Lakes, NJ).

Reagents. Cells were cultured in RPMI 1640, supplemented with $100 \mathrm{U} \mathrm{ml}^{-1}$ penicillin, $100 \mu \mathrm{g} \mathrm{ml}^{-1}$ streptomycin, $2 \mathrm{~mm} \mathrm{L-glutamine,}$ $5 \%$ fetal calf serum (all Invitrogen, Waltham, MA), and $50 \mu \mathrm{m}$ 2-mercaptoethanol (Sigma-Aldrich).

Antibodies. Fluorochrome-conjugated monoclonal antibodies to mouse antigens CD11b (M1/70), CD11c (N148), CD45 (30-F11), Integrin $\beta 7$ (FIB27), CD4 (RM4-5), CD8 $\alpha$ (53-6.7), CD19 (6D5), CD103 (2E7), CD62L (MEL-14), CD64 (X54-5/7.1), B220 (RA3-6B2), $\mathrm{T}$ cell receptor $\mathrm{V} \alpha 2$ (B20.1), CCR9 (CW-1.2), F4/80 (BM8), MHCII (M5/114.15.2), NRP-1 (3E12) and ST-2 (D1H9) were from Biolegend (San Diego, CA). FoxP3(150D/E4) was from eBiosciences (San Diego, CA).

Cell isolation. SI and colons were washed in Hank's balanced salt solution with $2 \%$ fetal calf serum, and the Peyer's patches were excised from the SI. The intestines were opened longitudinally and cut into $0.5 \mathrm{~cm}$ segments, which were incubated twice in Hank's balanced salt solution with 2 mм EDTA (Sigma-Aldrich) at $37^{\circ} \mathrm{C}$ with shaking for $20 \mathrm{~min}$. Supernatants were discarded and SI was digested with $1 \mathrm{mg} \mathrm{ml}^{-1}$ of collagenase VIII (Sigma-Aldrich) for $20 \mathrm{~min}$, while colons were digested with $0.85 \mathrm{mg} \mathrm{ml}^{-1}$ of collagenase V (Sigma-Aldrich), $1.25 \mathrm{mg} \mathrm{ml}^{-1}$ collagenase D (Roche, Penzberg, Ger), $1 \mathrm{mg} \mathrm{ml}^{-1}$ dispase (Gibco, Paisley, UK), and $30 \mu \mathrm{g} \mathrm{ml}^{-1} \mathrm{DNase}$ (Roche) for $40 \mathrm{~min}$. Cells 
were passed through a $40-\mu \mathrm{m}$ cell strainer (VWR, Radnor, PA) and stained for flow cytometry.

LNs were separated and incubated with 0.4 Wunsch units per ml of Liberase and $50 \mu \mathrm{g} \mathrm{ml}^{-1}$ of DNase (both Roche) for $45 \mathrm{~min}$ at $37^{\circ} \mathrm{C}$ while shaking. Single-cell suspensions were passed through a $40-\mu \mathrm{m}$ cell strainer and stained for flow cytometry.

Flow cytometry. Cell surface staining was performed in phosphatebuffered saline with $2 \%$ fetal calf serum and $10 \mathrm{~mm}$ EDTA for $30 \mathrm{~min}$ on ice. To determine proportions of live and dead cells, $20 \mu \mathrm{l}$ of 7AAD (Biolegend) was added to samples just before analysis, or $1 \mu \mathrm{l}$ of fixable viability dye eFluor 780 (eBioscience) was added to samples in phosphate-buffered saline for $30 \mathrm{~min}$ at $4{ }^{\circ} \mathrm{C}$ before staining with antibodies. Samples were acquired on an LSRII or a FACSAriaIII (both BD Biosciences) or sorted and analyzed on a FACSAriaIII. For intracellular staining, cells were stained for surface markers and washed. Cells were then fixed in $4 \%$ paraformaldehyde, permeabilized, and stained for intracellular transcription factors. Acquired data were analyzed using FlowJo software (version 9.2; Tree Star, Ashland, OR).

Proliferation assay. Twelve thousand and five hundred sorted 232-4 sMLN or cMLN CD $103^{+} \mathrm{CD}_{11 b^{-}}$DCs were cultured with $10^{5}$ CFSElabeled (Life Technologies, Carlsbad, CA) naive OT-I CD8 ${ }^{+}$T cells at $37^{\circ} \mathrm{C}$ for 3 days. CFSE dilution was assessed by flow cytometry.

Adoptive transfers. Cells were isolated from the MLNs and peripheral LNs of OT-II CD45.1 mice. Cells were labeled with CFSE for 15 min at $37^{\circ} \mathrm{C}$ and adoptively transferred intravenously in a CD45.2 host. Twenty-four hours later, the animal was gavaged with $10 \mathrm{mg} \mathrm{OVA}$ (grade IV, Sigma Aldrich). Proliferation was assessed by CFSE dilution.

Aldefluor assay. Aldehyde dehydrogenase activity was assessed using the aldefluor kit, according to the manufacturer's instructions (Stemcell Technologies, Manchester, UK). Briefly, cells were digested as described above and incubated with the aldefluor reagent or its inhibitor diethylaminobenzaldehyde. Cells were then washed and stained for flow cytometry.

RNA extraction. RNA was extracted using the MicroRNA kit (Qiagen, Venlo, The Netherlands) according to manufacturer's instructions. Contaminating genomic DNA was removed using the DNA-free kit (Qiagen). RNA was reversed transcribed using Superscript First Strand kit (Invitrogen).

Real-time quantitative PCR. cDNA was examined for the frequency of different transcripts using quantitative PCR. Reactions were performed using the Brilliant III Ultra Fast SYBR qPCR master mix (Aglient Technologies, Santa Clara, CA). Fluorescence levels were detected and analyzed using the 7900 HT Fast system (Applied Biosystems, Waltham, MA). Primers used were as follows: TBP forward: $5^{\prime}$-TCC ACA GCC TAT TCA GAA CAC C-3', reverse: 5'-CTA CTG CCT GCT GTT GTT GC-3'; GPR15 forward: 5' -CAT GAA TGT GAC TGG GCC CT- ${ }^{\prime}$, reverse: $5^{\prime}$-GTG CTG CTC CCA AAG TTG TG- $3^{\prime}$. Relative quantification was determined using the $\Delta \Delta \mathrm{Ct}$ method and normalized to the expression of the housekeeping gene.

Statistical analysis. For comparison of means between two groups, the data were analyzed using a Students $t$-test or a Mann-Whitney $U$ test. For comparisons involving more than two data sets, an analysis of variance was used. $P$-values of $<0.05$ were considered significant. All statistical analysis was performed using GraphPad Prism (GraphPad, San Diego, CA).

SUPPLEMENTARY MATERIAL is linked to the online version of the paper at http://www.nature.com/mi

\section{ACKNOWLEDGMENTS}

We thank Leo LeFrancois for the kind gift of 232-4 mice. We also thank the staff of the Central Research Facility for technical assistance, Cecilia Ansalone for assistance with Figure 19, and the University of Glasgow Flow
Cytometry Core Facility for assistance with cell sorting. S.A.H. was supported by a Capacity Building Award in Integrative Mammalian Biology funded by the BBSRC, BPS, KTN, MRC and SFC, AstraZeneca, GlaxoSmithKline, and Pfizer. V.C. was funded by a Medical Research Council award to SWFM MR/K021095/1; C.T. was funded by a Medical Research Council award to Professors Allan Mowat and Rob Nibbs MR/ L000598/1.

\section{DISCLOSURE}

The authors declared no conflict of interest.

c) 2016 Society for Mucosal Immunology

\section{REFERENCES}

1. Mowat, A.M. \& Agace, W.W. Regional specialization within the intestinal immune system. Nat. Rev. Immunol. 14, 667-685 (2014).

2. Belkaid, Y. \& Hand, T.W. Role of the microbiota in immunity and inflammation. Cell 157, 121-141 (2014).

3. Hooper, L.V. \& Macpherson, A.J. Immune adaptations that maintain homeostasis with the intestinal microbiota. Nat. Rev. Immunol. 10, 159-169 (2010).

4. Pabst, O. \& Mowat, A.M. Oral tolerance to food protein. Mucosal Immunol. 5, 232-239 (2012).

5. Svensson, M. et al. Retinoic acid receptor signaling levels and antigen dose regulate gut homing receptor expression on CD8 $+\mathrm{T}$ cells. Mucosal Immunol. 1, 38-48 (2008)

6. Kim, S.V. et al. GPR15-mediated homing controls immune homeostasis in the large intestine mucosa. Science 340, 1456-1459 (2013).

7. Jaensson, E. et al. Small intestinal CD103 + dendritic cells display unique functional properties that are conserved between mice and humans. J. Exp. Med. 205, 2139-2149 (2008).

8. Annacker, O. et al. Essential role for CD103 in the Tcell-mediated regulation of experimental colitis. J. Exp. Med. 202, 1051-1061 (2005).

9. Balic, A., Smith, K.A., Harcus, Y. \& Maizels, R.M. Dynamics of CD11C(+) dendritic cell subsets in lymph nodes draining the site of intestinal nematode infection. Immunol. Lett. 127, 68-75 (2009).

10. Bowcutt, R. et al. Heterogeneity across the murine small and large intestine. World J. Gastroenterol. 20, 15216-15232 (2014).

11. Carter, P.B. \& Collins, F.M. The route of enteric infection in normal mice. J. Exp. Med. 139, 1189-1203 (1974).

12. Tilney, N.L. Patterns of lymphatic drainage in the adult laboratory rat. J. Anat. 109, 14 (1971).

13. Van den Broeck, W., Derore, A. \& Simoens, P. Anatomy and nomenclature of murine lymph nodes: Descriptive study and nomenclatory standardization in BALB/cAnNCrl mice. J. Immunol. Methods 312, 12-19 (2006).

14. Laffont, S., Siddiqui, K.R. \& Powrie, F. Intestinal inflammation abrogates the tolerogenic properties of MLN CD103 + dendritic cells. Eur. J. Immunol. 40, 1877-1883 (2010)

15. Cerovic, V. et al. Lymph-borne CD8alpha dendritic cells are uniquely able to cross-prime CD8 T cells with antigen acquired from intestinal epithelial cells. Mucosal Immunol. 8, 38-48 (2015).

16. Tomura, M. et al. Monitoring cellular movement in vivo with photoconvertible fluorescence protein "Kaede" transgenic mice. Proc. Natl Acad. Sci. USA 105, 10871-10876 (2008).

17. Schmidt, T.H., Bannard, O., Gray, E.E. \& Cyster, J.G. CXCR4 promotes B cell egress from Peyer's patches. J. Exp. Med. 210, 1099-1107 (2013).

18. Cerovic, V. et al. Intestinal CD103(-) dendritic cells migrate in lymph and prime effector T cells. Mucosal Immunol. 6, 104-113 (2013).

19. Scott, C.L., Tfp, Z.M., Beckham, K.S., Douce, G. \& Mowat, A.M. Signal regulatory protein alpha (SIRPalpha) regulates the homeostasis of $\mathrm{CD103}(+) \mathrm{CD11b}(+) \mathrm{DCs}$ in the intestinal lamina propria. Eur. J. Immunol. 44, 3658-3668 (2014).

20. Jung, S. et al. Analysis of fractalkine receptor $\mathrm{CX}(3) \mathrm{CR} 1$ function by targeted deletion and green fluorescent protein reporter gene insertion. Mol. Cell. Biol. 20, 4106-4114 (2000).

21. Vezys, V., Olson, S. \& Lefrancois, L. Expression of intestine-specific antigen reveals novel pathways of CD8 T cell tolerance induction. Immunity $\mathbf{1 2}$, 505-514 (2000).

22. Denning, T.L. et al. Functional specializations of intestinal dendritic cell and macrophage subsets that control Th17 and regulatory Tcell responses are 


\section{ARTICLES}

dependent on the T cell/APC ratio, source of mouse strain, and regional Iocalization. J. Immunol. 187, 733-747 (2011).

23. Bruder, D. et al. Neuropilin-1: a surface marker of regulatory T cells. Eur. J. Immunol. 34, 623-630 (2004).

24. Schiering, C. et al. The alarmin IL-33 promotes regulatory T-cell function in the intestine. Nature 513, 564-568 (2014).

25. Sun, C.M. et al. Small intestine lamina propria dendritic cells promote de novo generation of Foxp3 T reg cells via retinoic acid. J. Exp. Med. 204, 1775-1785 (2007).

26. Coombes, J.L. et al. A functionally specialized population of mucosal CD103 + DCs induces Foxp3 + regulatory T cells via a TGF-beta and retinoic acid-dependent mechanism. J. Exp. Med. 204, 1757-1764 (2007).

27. Johansson-Lindbom, B. et al. Functional specialization of gut CD103+ dendritic cells in the regulation of tissue-selective T cell homing. J. Exp. Med. 202, 1063-1073 (2005).

28. Scott, C.L. et al. CCR2 + CD103 + intestinal dendritic cells develop from DC-committed precursors and induce interleukin-17 production by Tcells. Mucosal Immunol. 8, 327-339 (2015).
29. Rivollier, A., He, J., Kole, A., Valatas, V. \& Kelsall, B.L. Inflammation switches the differentiation program of Ly6Chi monocytes from antiinflammatory macrophages to inflammatory dendritic cells in the colon. J. Exp. Med. 209, 139-155 (2012).

30. Persson, E.K. et al. IRF4 transcription-factor-dependent CD103(+) $\mathrm{CD} 11 \mathrm{~b}(+)$ dendritic cells drive mucosal T helper 17 cell differentiation. Immunity 38, 958-969 (2013).

31. Kamath, A.T., Henri, S., Battye, F., Tough, D.F. \& Shortman, K. Developmental kinetics and lifespan of dendritic cells in mouse lymphoid organs. Blood 100, 1734-1741 (2002).

32. Diehl, G.E. et al. Microbiota restricts trafficking of bacteria to mesenteric lymph nodes by CX(3)CR1(hi) cells. Nature 494, 116-120 (2013).

33. Chirdo, F.G., Millington, O.R., Beacock-Sharp, H. \& Mowat, A.M. Immunomodulatory dendritic cells in intestinal lamina propria. Eur. J. Immunol. 35, 1831-1840 (2005).

34. Yang, X.O. et al. Molecular antagonism and plasticity of regulatory and inflammatory T cell programs. Immunity 29, 44-56 (2008).

35. Nguyen, L.P. et al. Role and species-specific expression of colon T cell homing receptor GPR15 in colitis. Nat. Immunol. 16, 207-213 (2015). 